\title{
Design of a Flexible Minkowski-Like Pre-Fractal (MLPF) antenna with Different Ground Planes for VHF LMR
}

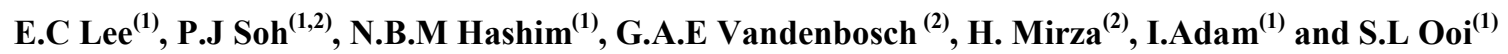 \\ (1) School of Computer and Communication Engineering, Universiti Malaysia Perlis (UniMAP), 02000 Kuala Perlis, \\ Perlis, MALAYSIA \\ Email:eclee_tiger@yahoo.com,[pjsoh,baya,ismahayati]@unimap.edu.my,sooliam@gmail.com
}

(2)ESAT-TELEMIC, Katholieke Universiteit Leuven, 3001 Leuven, BELGIUM

Email:[pingjack.soh, hidayath.mirza, guy.vandenbosch]@esat.kuleuven.be

\begin{abstract}
This work investigates the suitability of a flexible Minkowski-like pre-fractal antenna (MLPF) for application in the Very High Frequency (VHF) Land and Mobile Radio (LMR) frequency bands (136-174 MHz). The proposed center-fed, third iteration antenna has been manufactured using both copper tape and ShieldIt Super, a conductive textile. It has been tested on four types of ground plane, i.e. rectangular, fractal Minkowski, meander line and L-shaped. It is observed that an L-shaped ground plane performs the best, and provides an additional frequency tuning parameter to the overall structure. Simulated and measured $S_{11}$ show a good agreement and the proposed antenna is working within the desired frequency range. A simulated gain and efficiency of more than $1.9 \mathrm{~dB}$ and $76 \%$, respectively, are obtained. The measurement in a Satimo SG64 anechoic chamber provided $0 \mathrm{~dB}$ gain and about $48 \%$ efficiency.
\end{abstract}

\section{INTRODUCTION}

Body-worn antennas have been receiving a growing interest in recent years. Unlike others portable antennas, which are usually attached to some equipment, they are attached to the human body, which produces several challenges. Few researches have been dedicated to the development of wearable antennas that operate in the Very High Frequency (VHF) band. Concerns of health hazards when used in proximity of the body, detuning and resonance shifting, material suitability and subjectivity of antenna performance to users' physical traits have also more or less hampered the development of more flexible antennas for this application. Recognizing the importance of the ground plane in effectively reducing any detuning and resonance shifting, the purpose of this work is to investigate the suitability of several ground plane structures in the case of a flexible antenna.

Classic fractal antennas, such as the Sierpinski gasket fractal, the Hilbert curve, the Koch curves have been successfully implemented to provide multi-band resonances in conventional narrowband structures. This is thanks to self-similarity. Miniaturization in size (more than 70\% [1,2]) and bandwidth enlargement of up to $17 \%$ [1] also came with the use of such concept at the expense of overall gain. However, the ability to achieve an omni-directional azimuth pattern, enabling signal reception/transmission from and to all directions is deemed more important in wearable systems.

One of the earlier works which investigates the use of a wearable antenna at this frequency is a research on wearable FM radio antennas [3]. Four antenna designs have been considered, and a wide dipole antenna was found to be the best performing, obtaining a maximum gain of $-13 \mathrm{dBi}$, depending on the posture of the user. Bowtie and spiral topologies made from conductive nylon and copper coated fabric, operating above $250 \mathrm{MHz}$ have also been investigated in [4]. The mentioned work also found that the separation between antenna and users' body is a critical parameter in determining the reflection coefficient $\left(S_{11}\right)$ and bandwidth stability. This effect could be reduced by introducing a larger ground plane, which unfortunately is difficult to be incorporated into already large antennas at low operating frequencies (between 100-250 MHz) [5]. Full azimuth coverage required multi-element antennas to be used, which possibly will results in a larger antenna size. Thus, a Minkowski-like Pre-Fractal (MPLF) antenna is chosen as the basis in this work, Due to the presence of the slits, which increase the current path length, it is easy to miniaturize. The resulting size of the MPLF makes it suitable only to be placed on the back of users. Moreover, investigations in [6] which indicated the achievement of higher gain and efficiency at this location, add to the many advantages of this antenna topology.

\section{ANTENNA DESIGN}

The proposed third iteration MLPF antenna is designed based on an iteration length, $L_{\mathrm{n}}$. It is calculated as follow [1]: 


$$
L_{n}=2 L_{n+1}+W_{1}^{n+1}+2 W_{2}^{n+1}
$$

where $n$ is the iteration number, $W_{1}{ }^{\mathrm{n}+1}=a_{1} L_{\mathrm{n}}$; is the width of the middle segment, and $W_{2}{ }^{\mathrm{n}+1}=a_{2} L_{\mathrm{n}}$; is the indentation width. Parameters $a_{1}$ and $a_{2}$, which determine the efficiency of the size reduction, are ratios of middle segment width, and indentation width, both with respect to the iteration length $\left(L_{\mathrm{n}}\right)$.

Suitable values for both ratios have to be determined to avoid higher order elements from detaching from their preceding elements when $a_{1}>a_{2}$, or to avoid overlapping of elements from different iteration orders when $a_{1}=a_{2}$. Two materials, i.e a thin flexible copper tape and ShieldIt Super - a conductive fabric from LessEMF Inc USA - were used. Simulations were done using the commercial electromagnetic solver CST Microwave Studio. The antenna was designed for LMR applications, i.e. for a lowest operating frequency of $136 \mathrm{MHz}$. The copper tape used has a thickness $t$ of $0.035 \mathrm{~mm}$, and a conductivity $\sigma$ of $5.8 \times 10^{7} \mathrm{~S} / \mathrm{m}$, while ShieldIt fabric has $t=0.17 \mathrm{~mm}$ and $\sigma=6.5 \times 10^{4} \mathrm{~S} / \mathrm{m}$. Conversion of the surface resistance $R_{\mathrm{s}}$ to conductivity $\sigma$ has been carried out using $\sigma=1 /\left(R_{\mathrm{S}} \mathrm{x} t\right)$ [7]. A Minkowski antenna with $a_{1}=0.03, a_{2}=0.15$ is deemed the most suitable to produce the lowest desired frequency of $136 \mathrm{MHz}$.

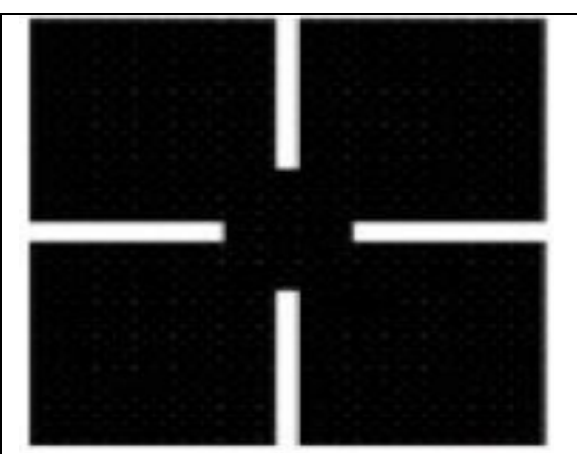

(a)

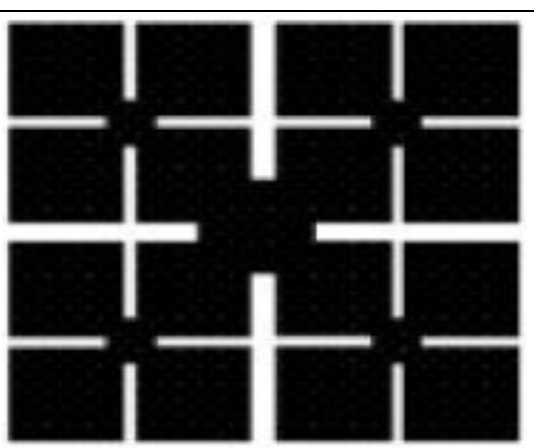

(b)

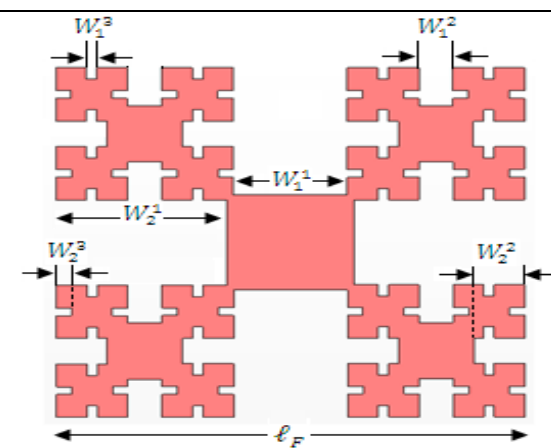

(c)

Fig. 1: Minkowski like Pre-Fractal (MLPF) structures: (a) First iteration, (b) second iteration, (c) Proposed third iteration MLPF [1]

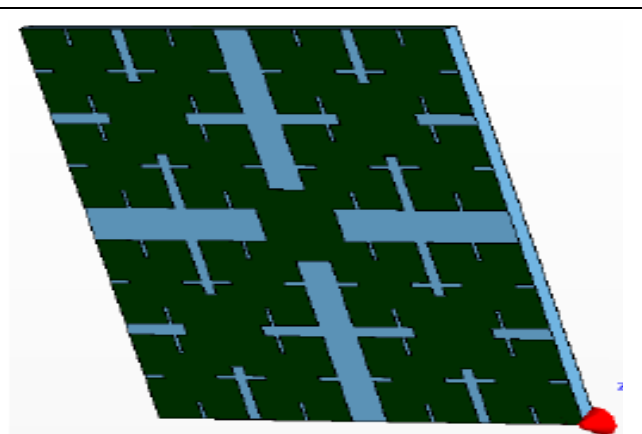

(a)

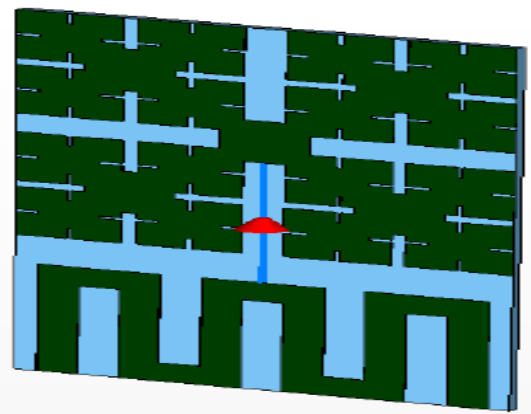

(c)

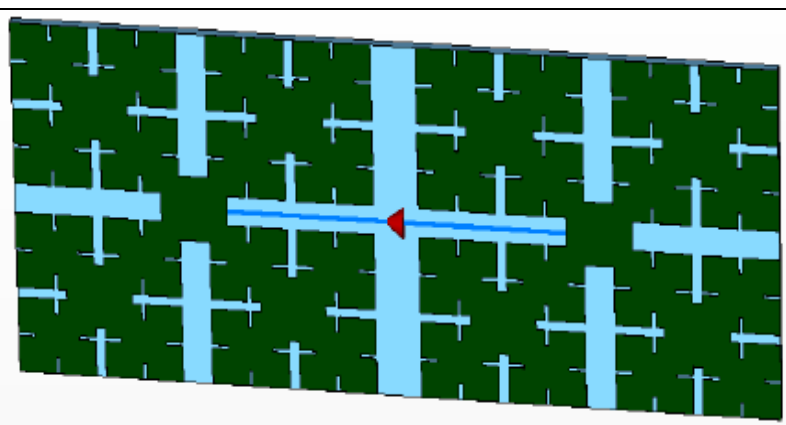

(b)

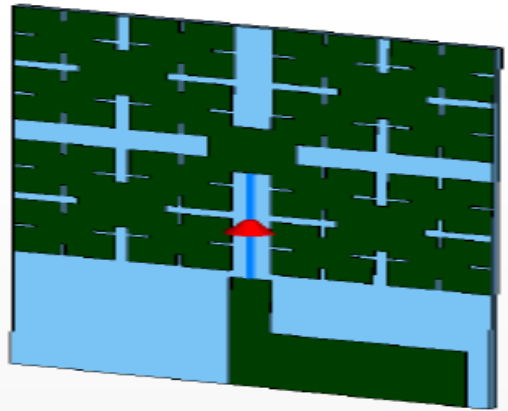

(d)

Fig. 2: Investigated ground plane structures for the proposed third order Minkowski array: (a) Square (b) Minkowski (c) meander line (d) L-shaped 


\section{RESULTS AND DISCUSSION}

The first ground plane, a $448.5 \times 448.5 \mathrm{~mm}$ ground plane is placed directly behind the radiator and fed at the corner of the MLPF; see Fig. 2(a). A sharp and narrowband resonance is produced with $S_{11}=-9.8 \mathrm{~dB}$ at a frequency of 202.4 MHz. In the desired band between 136-174 MHz, only about $-1 \mathrm{~dB} S_{11}$ was produced, with a gain of less than $-2.4 \mathrm{~dB}$. The second ground plane, which is a similarly shaped and sized MLPF of the third order, is printed on the same side as the radiating element, as shown in Fig. 2(b). This is in order to create two similarly shaped elements, one of them electrically grounded, to emulate a dipole. The resulting performance is marginal, producing about $-2.5 \mathrm{~dB}$ of $S_{11}$ within the operating frequency band. On the other hand, the efficiency has improved up to $32 \%$ to $43 \%$, while the simulated gains are in between 2.0 and $2.5 \mathrm{~dB}$, see Table 1 . The next ground plane is a three turn meander line, which is printed on the same side, $50 \mathrm{~mm}$ from the radiating element. This structure is shown in Fig 2(c). It produced the best $S_{11}$ of -3.2 $\mathrm{dB}$ and a maximum gain of $2.2 \mathrm{~dB}$, within the desired band. A dipole-like radiation pattern was produced by this structure in the elevation plane. It also exhibits a higher efficiency of about $50 \%$. Finally, an L-shaped ground plane which is printed $10 \mathrm{~mm}$ away from the antenna is investigated, as shown in Fig. 2(d). An $S_{11}$ of $-12 \mathrm{~dB}$ is observed, while a minimum simulated gain and efficiency of $1.9 \mathrm{~dB}$ and $76 \%$, respectively, are achieved within the operating frequency band.

For the fabricated prototype, simulated and measured $S_{11}$ are shown in Fig 3. The measured $S_{11}$ shows a small degradation in comparison to the simulation, producing $-9 \mathrm{~dB}$ at best. The measured efficiency and gain, obtained in a Satimo SG64 anechoic chamber, are about $48 \%$ and $0 \mathrm{~dB}$, respectively. This is probably due to the interconnection losses. Since a single piece of copper tape with such large dimension is practically unrealistic, smaller pieces have to be joined together through soldering. An overview of the simulated and measured properties of the antenna prototypes are given in Table 1. Simulated and measured radiation patterns are given in Fig. 4. The agreement is fair.

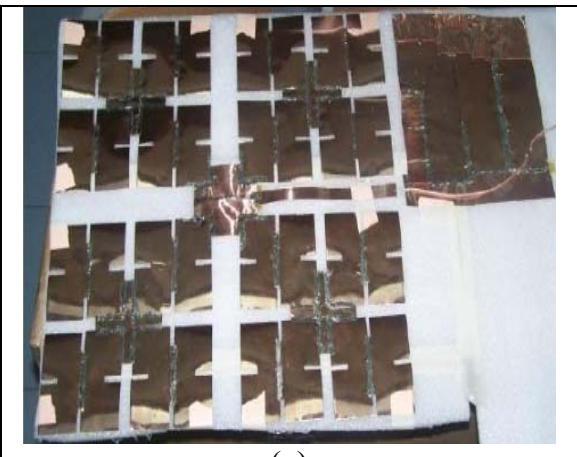

(a)

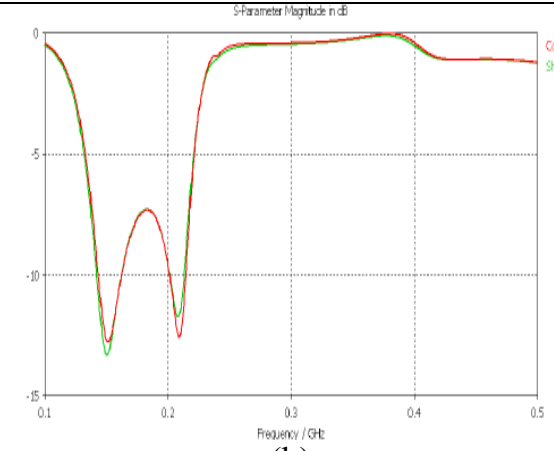

(b)

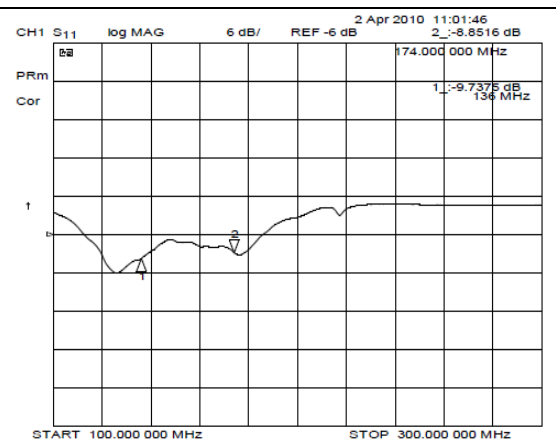

(c)

Fig. 3: Measurement of the proposed antenna: (a) fabricated prototype using copper tape; (b) comparison of simulations with L-shaped ground plane using copper tape and ShieldIt Super; (c) measurement result using vector network analyzer

Tab. 1 Simulation and measured S11, gain and efficiency of the MLPF antenna

\begin{tabular}{|c|c|c|c|c|c|c|}
\hline \multicolumn{2}{|c|}{ Freq/ Ground plane type } & Square (Sim) & Minkowski(Sim) & Meanderline(Sim) & L shaped(Sim) & L-shaped(Meas) \\
\hline \multirow[t]{3}{*}{$136 \mathrm{MHz}$} & $\mathrm{S} 11(\mathrm{~dB})$ & -0.33 & -1.73 & -3.00 & -6.17 & -9.74 \\
\hline & Efficiency (\%) & 0.54 & 32.26 & 47.27 & 75.46 & 26.55 \\
\hline & Gain $(\mathrm{dB})$ & -8.52 & 2.06 & 1.73 & 1.94 & -2.84 \\
\hline \multirow[t]{3}{*}{$151 \mathrm{MHz}$} & $\mathrm{S} 11(\mathrm{~dB})$ & -0.34 & $\begin{array}{ll}-1.90 \\
\end{array}$ & -2.73 & -12.75 & \\
\hline & Efficiency (\%) & 1.28 & 34.84 & 44.04 & 94.63 & 31.33 \\
\hline & Gain $(\mathrm{dB})$ & -5.04 & 2.20 & 1.81 & 2.03 & -2.01 \\
\hline \multirow[t]{3}{*}{$162 \mathrm{MHz}$} & $\mathrm{S} 11(\mathrm{~dB})$ & -0.60 & -2.17 & -2.64 & -10.13 & \\
\hline & Efficiency (\%) & 2.56 & 38.73 & 45.93 & 90.36 & 40.83 \\
\hline & Gain $(\mathrm{dB})$ & -4.09 & 2.34 & 2.16 & 2.10 & -0.91 \\
\hline \multirow[t]{3}{*}{$174 \mathrm{MHz}$} & $\mathrm{S} 11(\mathrm{~dB})$ & -0.99 & -2.53 & -3.22 & -7.82 & -8.85 \\
\hline & Efficiency (\%) & 5.75 & 43.18 & 50.12 & 83.55 & 47.86 \\
\hline & Gain $(\mathrm{dB})$ & -2.43 & 2.45 & 2.02 & 2.17 & -0.57 \\
\hline
\end{tabular}




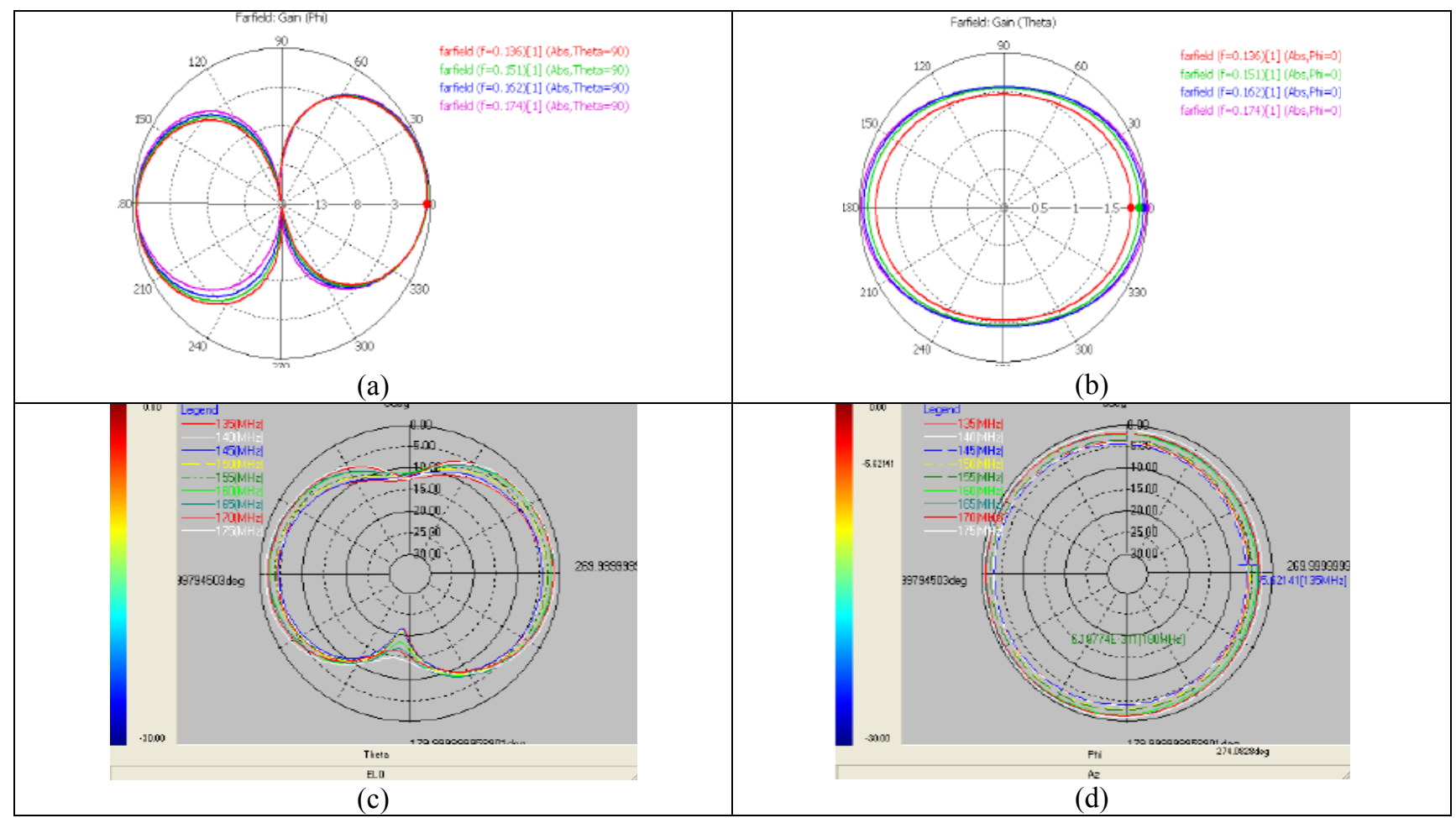

Fig. 4: Radiation patterns: simulated using CST (a) azimuth plane (b) elevation plane, and measured (c) azimuth plane (d) elevation plane.

\section{CONCLUSION}

Minkowski like Pre-Fractal antennas with different ground plane types are designed and discussed. Simulation results indicate that the L-shaped ground plane prototype produces the best performance when placed on the same face as the radiator, $10 \mathrm{~mm}$ away. Measurement results prove that the antenna is working in the desired frequency band between 136 and $174 \mathrm{MHz}$ with an omni-directional radiation pattern. Gain and efficiency are about $0 \mathrm{~dB}$ and $48 \%$ with a $S_{11}$ of less than $-6 \mathrm{~dB}$.

\section{REFERENCES}

[1] M. Tayefeh and K. Aligodarz, "Wideband Miniaturized L-Probe Fed Fractal Clover Leaf Microstrip Patch Antenna," in 13th International Symposium on Antennas (JINA), Nice, France, 2004.

[2] C. Wen-Ling, W. Guang-Ming, and Z. Chen-Xin, "Small-Size Microstrip Patch Antennas Combining Koch and Sierpinski Fractal-Shapes," Antennas and Wireless Propagation Letters, IEEE, vol. 7, pp. 738-741, 2008.

[3] T. Kellomaki, J. Heikkinen, and M. Kivikoski, "Wearable antennas for FM reception," in Antennas and Propagation, 2006. EuCAP 2006. First European Conference on, 2006, pp. 1-6.

[4] J. C. G. Matthew, B. Pirollo, A. Tyler, and G. Pettitt, "Body wearable antennas for UHF/VHF," in Antennas and Propagation Conference, 2008. LAPC 2008. Loughborough, 2008, pp. 357-360.

[5] J. C. G. Matthews and G. Pettitt, "Development of flexible, wearable antennas," in Antennas and Propagation, 2009. EuCAP 2009. 3rd European Conference on, 2009, pp. 273-277.

[6] P. J. Soh, G. A. E. Vandenbosch, V. Volski, and H. M. R. Nurul, "Characterization of a Simple Broadband Textile Planar Inverted-F Antenna (PIFA) for on Body Communications," in Applied Electromagnetics and Communications, 2010. ICECom 2010. 20th International Conference on Dubrovnik, Croatia, 2010.

[7] N. H. M. Rais, P. J. Soh, F. Malek, S. Ahmad, N. B. M. Hashim, and P. S. Hall, "A review of wearable antenna," in Antennas \& Propagation Conference, 2009. LAPC 2009. Loughborough, 2009, pp. 225-228. 